J. Lake Sci. (湖泊科学), 2017, 29(1): 22-31

DOI 10. 18307/2017. 0103

(c) 2017 by Journal of Lake Sciences

\title{
太湖水龄分布特征及“引江济太”工程对其的影响”
}

\author{
黄春琳 $^{1}$, 李 熙 $^{1 * *}$, 孙永远 $^{2}$ \\ (1: 河海大学海岸灾害及防护教育部重点实验室,南京 210098) \\ $(2$ : 江苏省水文水资源勘测局, 南京 210029$)$
}

摘 要: 太湖作为典型的风生流湖泊, 风场对水体运输和交换的过程起着重要的作用. 基于环境水动力学模型 EFDC 源 程序建立了染色剂模型以及水龄模型, 借助水龄研究太湖水体的长期输运过程和更新速率特征, 从而为太湖的调水工程 管理提供科学依据. 本文在研究春、夏、秋、冬季不同风场作用下太湖的水龄季节性分布特征的基础上, 结合太湖实测风 速、流量数据及 “引江济太” 工程调水运行的现状, 着重分析了望虞河枢纽调水运行以及新沟河工程对太湖水龄分布的影 响. 数值试验的模拟结果表明: 太湖水体交换受季风影响明显, 春、夏季的水龄相对较大、水体交换较差; 靠近湖流人口处 的地方水龄较小, 远离人口的地方水龄较大; 水流流向与风向一致时水龄减小, 水循环加快, 反之则减慢. 望虞河引水引 工程能够减小贡湖及湖心区的水龄, 加快贡湖湖区及湖心区的水循环; 新沟河工程引水能够减小梅梁湖区的水龄, 改善 该湖区的水质. 引水工程的实施对加快整个太湖的水循环做出重要贡献.

关键词: 太湖; 风场; 染色剂; 水龄;EFDC 模型

\section{Water age distribution of the Lake Taihu and impact of the Yangtze River to Lake Taihu Water Transfer Project on the water age}

\author{
HUANG Chunlin ${ }^{1}$, LI Xi ${ }^{1 * *} \&$ SUN Yongyuan ${ }^{2}$ \\ (1: Key Laboratory of Coastal Disaster and Defence, Minstry of Education, Hohai University, Nanjing 210098, P.R.China) \\ (2: Jiangsu Province Hydrology and Water Resource Investigation Bureau, Nangjing 210029, P.R.China)
}

\begin{abstract}
Lake Taihu is a typical wind-driven lake, and wind plays an important role in process of water exchange and concentration transportation. Based on Environmental Fluid Dynamics Model (EFDC), water age model and conservative dye model were set up to study characteristics of seasonal water age distribution and to further reveal the impacts of the Wangyu River Water Transfer Project and the Xingou River to the Lake Taihu Transfer Project on water age distribution on basis of winds, inflow and outflow, operations of existing water transfer projects. It is concluded that by the numerical test results, water exchange of Lake Taihu is mainly influenced by the monsoons. Water age is comparably larger in the spring and summer, which indicates the water exchange rate in the spring and summer is lower than that in the autumn and winter. Water ages near inflow locations are comparably smaller, and the locations far from the inflows are larger. Water age is smaller when the flow direction is the same as wind direction, while the exchange of water is increased at the same time and on the contrary water exchange is reduced. During operation of the Wangyu River Water Transfer Project, the water ages of the Gonghu Bay and Middle Lake Taihu are basically reduced, and water exchange is increased in the areas. During operation of the Xingou River Water Transfer Project, the water ages of the Meiliang Bay are basically reduced, and water quality is improved in the area. The water exchange of Lake Taihu will be significantly improved with continuous or occasional transfer operations.
\end{abstract}

Keywords: Lake Taihu; wind; dye; water age; Environmental Fluid Dynamics Model

人湖流量、风场等在湖体的水体运动过程中起很重要的作用, 三维环境流体动力学模型 EFDC 提供了一

* 江苏省水利科技项目 (2015054) 和江西省水利科技项目(KT201405) 联合资助. 2016-06-06 收稿; 2016-07-03 收修改稿. 黄春琳(1992 ), 女, 硕士研究生;E-mail:805282052@ qq.com.

** 通信作者;E-mail:xili@ hhu.edu.cn. 
个高分辨率的工具来研究太湖的水体交换. 水龄可以定量反映水体的运动和交换程度以及滞留情况,对评 估水质的变化具有重要意义. Deleersnijder 等 ${ }^{[1]}$ 介绍了基于对流-扩散输运方程的水龄理论,这个新的水龄 定义提供了一个健全的方法来定量地研究欧拉坐标框架下的物质溶解的输运特征. $\mathrm{Li}$ 等 ${ }^{[2]}$ 运用 EFDC 三维 水动力模型, 借助水龄的定义, 研究了“引引江济太”引水量对太湖水循环的影响.

太湖作为中国的第三大淡水湖, 由于富营养化而严重影响了饮用水的安全, 政府部门近年来采取了许 多措施来提升太湖水质, 包括建更多的污水处理厂, 禁止使用含磷清洁剂等. 此外, 一个重要的生态工 程——“江济太”工程在 2002 年启动, “引江济太” 工程将更优质的长江水通过望虞河引人太湖, 最终从太 浦河流出, 以期通过此举来加快太湖的水循环, 改善太湖的水质状况 ${ }^{[3-5]}$. 此外, 在 2014 年太湖北部流域内 开始疏通新沟河、新孟河两条河道,太湖将通过新孟河、望虞河补水,借助走马塘、新沟河、太浦河向长江排 水,形成 “两进三出” 的循环系统, 通过让水体动起来改善水质. 本文基于 EFDC 三维水动力模型, 建立水龄 模型来研究太湖水体交换速率. 基于实测径流、风场数据、引水工程的调水运行数据, 对太湖进行三维水动 力模拟, 研究风场作用下太湖水龄分布的季节性特征以及“引江济太”工程对太湖水龄分布特征的影响.

\section{1 研究区域}

太湖 ( $30^{\circ} 55^{\prime} 40^{\prime \prime} \sim 31^{\circ} 32^{\prime} 58^{\prime \prime} \mathrm{N}, 119^{\circ} 52^{\prime} 32^{\prime \prime} \sim 120^{\circ} 36^{\prime} 10^{\prime \prime} \mathrm{E}$ ) 横跨江、浙两省, 西和西南侧为丘陵山地, 东侧 以平原和水网为主. 地处亚热带, 气候温和湿润, 属季风气候, 夏季受热带海洋气团影响, 盛行东南季风, 温 和多雨; 冬季受北方高压气团控制, 盛行偏北季风, 寒冷干燥. 太湖是典型的风生流湖泊, 且水深极浅, 湖域 面积几乎全年不变. 太湖水域面积为 $2338.1 \mathrm{~km}^{2}$, 平均年出湖径流量 $75 \times 10^{8} \mathrm{~m}^{3}$, 蓄水量 $44 \times 10^{8} \mathrm{~m}^{3}$, 年降水 量 $1100 \sim 1150 \mathrm{~mm}$, 年平均气温 $16 \sim 18^{\circ} \mathrm{C}$, 平均水深 $1.89 \mathrm{~m}$, 最大水深 $2.6 \mathrm{~m}$, 年平均风速 $3.5 \sim 5.0 \mathrm{~m} / \mathrm{s}$. 为分 析“引江济太” 工程建设对太湖水龄的影响, 环太湖共采集 12 个站点进行分析对比, 其中 $1^{\#} 、 5^{\#} \sim 7^{\#}$ 站点位于 引水口, $1^{\#} \sim 4^{\#} 、 12^{\#}$ 站点位于取水口, $2^{\#} 、 10^{\#}$ 站点为水位验证站, 其余站点为流量控制点 (图 1).

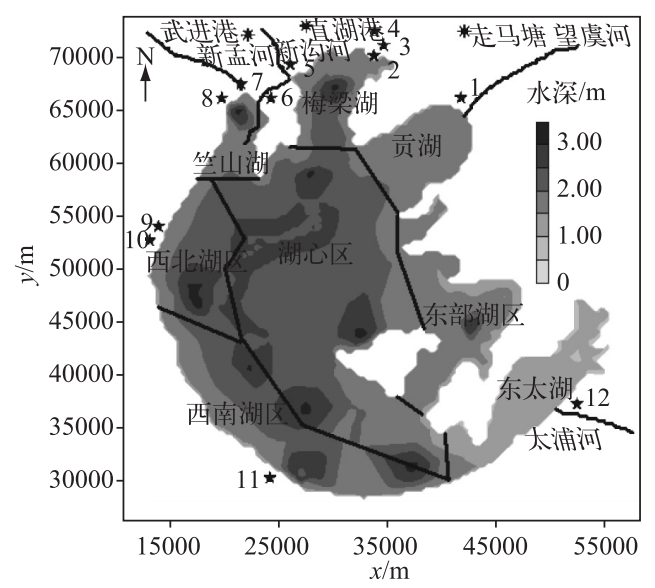

图 1 太湖地形及引水工程示意 $(1$ : 望亭; 2 : 犊山闸; 3 : 梅梁湖泵站; 4 : 大渲河原站; 5 :龚巷桥;

6 : 雅浦桥; 7 : 百渎口; 8 : 浯溪桥; 9 : 城东港; 10 : 大浦口; 11 : 长兒港; 12 : 太浦)

Fig.1 Bathymetry and sketch for water transfer layout of Lake Taihu

\section{2 数值模型}

\subsection{EFDC 三维水动力模型}

2.1.1 三维水动力模型的建立 EFDC 模型是一个多参数的有限差分模型, 可用于河流、湖泊、水库、湿地系 统、河口和海洋等水体的水动力学和水质模拟 ${ }^{[6]}$. 本文将其应用于太湖的研究. 控制方程在水平方向上采用 笛卡尔直角坐标系或者曲线正交坐标系,垂向采用 Sigma 坐标. 在 Sigma 坐标系下 EFDC 模型的连续性方程 
和动量方程为 ${ }^{[7-9]}$ :

$$
\begin{gathered}
\partial_{t}(m H)+\partial_{x}\left(m_{y} H u\right)+\partial_{y}\left(m_{x} H v\right)+\partial_{z}(m \omega)=Q_{H} \\
\partial_{t}(m H u)+\partial_{x}\left(m_{y} H u u\right)+\partial_{y}\left(m_{x} H v u\right)+\partial_{z}(m w u)-\left(m f-u \partial_{y} m_{x}+v \partial_{x} m_{y}\right) H v= \\
-m_{y} H \partial_{x}(g \zeta+p)-m_{y}\left(\partial_{x} h-z \partial_{x} H\right) \partial_{z} p+\partial_{z}\left(m \frac{A_{v}}{H} \partial_{z} u\right)+Q_{u} \\
\partial_{t}(m H v)+\partial_{x}\left(m_{y} H u v\right)+\partial_{y}\left(m_{x} H v v\right)+\partial_{z}(m w v)+\left(m f-u \partial_{y} m_{x}+v \partial_{x} m_{y}\right) H u= \\
-m_{x} H \partial_{y}(g \zeta+p)-m_{x}\left(\partial_{y} h-z \partial_{y} H\right) \partial_{z} p+\partial_{z}\left(m \frac{A_{v}}{H} \partial_{z} v\right)+Q_{v} \\
\left(\tau_{x z}, \tau_{y z}\right)=A_{v} H^{-1} \partial_{z}(u, v) \\
\partial_{z} p=-g H b=-g H\left(\rho-\rho_{0}\right) \rho_{0}^{-1} \\
\left(\tau_{x z}, \tau_{y z}\right)=\left(\tau_{s x}, \tau_{s y}\right)=c_{\mathrm{s}}\left(U_{\mathrm{w}}^{2}+V_{\mathrm{w}}^{2}\right)^{1 / 2}\left(U_{\mathrm{w}}, V_{\mathrm{w}}\right)
\end{gathered}
$$

式中, $x 、 y$ 为水平笛卡尔坐标系; $z$ 为坚向 Sigma 坐标; $H$ 为水深, $\zeta$ 是水面高程, $\eta$ 为相对水位, 定义 $\zeta=H+$ $\eta ; u 、 v 、 w$ 分别为 $x 、 y 、 z$ 方向的流速; $Q_{H}$ 为体积源项,包括降雨、蒸发、渗流; $Q_{u} 、 Q_{v}$ 为 $x 、 y$ 方向的动量源项; $p$ 为附加静水压力; $f$ 为柯氏参数; $\tau_{x z} 、 \tau_{y z}$ 为 $x 、 y$ 方向的垂向剪切应力; $A_{v}$ 为垂向湍流动量扩散系数; $\rho$ 和 $\rho_{0}$ 分别为实际和参考密度, $b$ 为浮力. $m_{x} 、 m_{y}$ 为水平坐标因子, 且 $m=m_{x} m_{y} . U_{\mathrm{w}}$ 和 $V_{\mathrm{w}}$ 为水平面 $10 \mathrm{~m}$ 高度处风 速 $x$ 和 $y$ 方向的分量, 风应力系数为:

$$
c_{\mathrm{s}}=0.001 \frac{\rho_{\mathrm{a}}}{\rho_{\mathrm{w}}}\left[0.8+0.065\left(U_{\mathrm{w}}^{2}+V_{\mathrm{w}}^{2}\right)^{1 / 2}\right]
$$

式中, 风速单位为 $\mathrm{m} / \mathrm{s}, \rho_{\mathrm{a}}$ 和 $\rho_{\mathrm{w}}$ 分别代表空气和水的密度.

2.1 .2 模型主要计算参数以及模型的率定 本文采用 2014 年太湖的流量、气象、水位以及地形资料, 基于 EFDC 建立三维水动力模型, EFDC 模型的应用已十分成熟, 时间步长通过 CFL (科朗数) 稳定条件确定. 鉌 率与水深、床面形态等因素有关, 根据计算率定. 紊动粘性系数采用 Smagorinsky 公式计, $C_{\mathrm{s}}$ 为 Smagorinsky 系数,通常取 $0.28^{[8]}$. 数学模型的初始条件一般分热启动和冷启动两种. 前者一般通过计算得到计算区域内 相对准确的水位、流速场, 后者则假定一种均匀分布, 与实际情况不一定相符, 但通过边界信息的传人, 误差 会逐渐消失; 本文采用了冷启动. 数学模型的边界条件包括开边界 (水边界) 和固定边界 (岸边界). 因人湖 河流的断面尺度相对于湖域面积很小, 故本文的计算将太湖视为一个封闭的区域, 人湖河流的流量通过源 汇项输人. 模型网格采用正交曲线网格, 最大网格尺度 $1000 \mathrm{~m}$, 最小网格 $800 \mathrm{~m}$, 网格数 15625 个, 垂向分为 4 层, 每层的厚度由湖底地形以及水面高程来决定. 模型主要受径流、表层风应力的驱动, 温度在模型中视为 常数. 模型的率定通过验证站的实测水位进行, 选取犊山闸和大浦口站的水位进行模型的率定, 模拟结果与 实测值吻合较好, 表明模型精度满足要求 (图 2). 由于实际情况下太湖的换水周期为 $300 \mathrm{~d}$ 左右 ${ }^{[3]}$, 本文采 用的流量边界基本接近实际情况,因此模拟周期为 $365 \mathrm{~d}$.
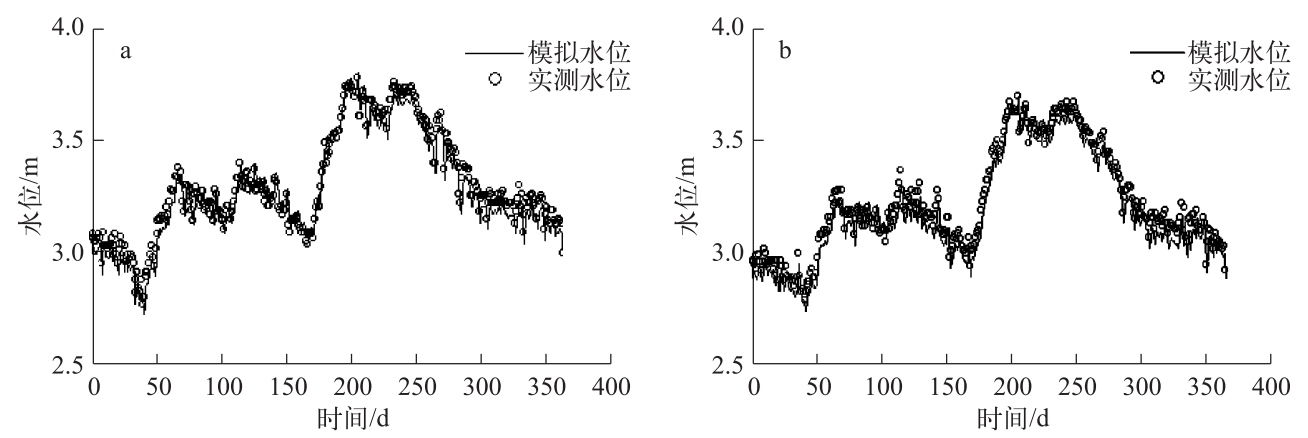

图 2 犊山闸 (a) 和大浦口 (b) 计算水位和实测水位的对比验证

Fig.2 Comparison of the measured and simulated water levels in Dushan Gate (a) and Dapukou (b) sites 


\section{2 水龄的定义}

水龄被定义为某个固定的水质点从人口传输到某个指定点所用的时间, 通常人口处的水龄设为 0 . 水龄 可以通过系统中示踪剂的浓度分布以及一个虚拟的水龄浓度来计算. 假设系统中只有一种示踪剂, 那么示 踪剂浓度和水龄浓度满足以下两个控制方程 ${ }^{[10-16]}$ :

$$
\begin{gathered}
\frac{\partial c(t, \vec{x})}{\partial t}+\nabla(\vec{u} c(t, \vec{x})-\vec{K} \nabla c(t, \vec{x}))=0 \\
\frac{\partial \alpha(t, \vec{x})}{\partial t}+\nabla(\vec{u} \alpha(t, \vec{x})-\vec{K} \nabla \alpha(t, \vec{x}))=c(t, \vec{x})
\end{gathered}
$$

式中, $c$ 为示踪剂浓度, $\vec{u}$ 为速度场, $\vec{K}$ 为扩散张量, $t$ 为时间, $\vec{x}$ 为空间坐标. 平均水龄 $a$ 可由下式计算:

$$
a(t, \vec{x})=\frac{\int_{0}^{\infty} \tau c \mathrm{~d} \tau}{\int_{0}^{\infty} c \mathrm{~d} \tau}=\frac{\alpha(t, x)}{c(t, x)}
$$

基于 EFDC 的源程序,增加了水龄的数值模块. 为了率定风场作用下的水龄, 与 $\mathrm{Li}$ 等 ${ }^{[2]}$ 的计算结果进行 对比率定, 在 $\mathrm{SE} 、 \mathrm{~N}$ 风向下假设单人口流量下各测站水龄计算结果拟合结果良好 (表 1), 因此本文计算的风 场下水龄的精度满足要求.

表 1 本文水龄结果与 $\mathrm{Li}$ 等 2011 年水龄计算结果 ${ }^{[2]}$ 对比

Tab.1 Comparison of water ages between present calculation and Li et al (2011) ${ }^{[2]}$

\begin{tabular}{ccc}
\hline 监测点 (风向 $)$ & $\mathrm{Li}$ 等 2011 年计算的水龄 $/ \mathrm{d}$ & 本文计算的水龄/d \\
\hline $\mathrm{B}(\mathrm{N})$ & 310 & 308 \\
$\mathrm{~B}(\mathrm{SE})$ & 300 & 295 \\
$\mathrm{C}(\mathrm{N})$ & 300 & 300 \\
$\mathrm{C}(\mathrm{SE})$ & 200 & 194 \\
\hline
\end{tabular}

\section{3 计算方案}

本文的计算将太湖视为一个封闭的区域,太湖的环湖河道众多,但其流量均较小, 因此模型在设置流量 边界时, 考虑上游主要人湖河流有从西苕溪水系流人的城东港、大渲河原站、百渎口和长㝸港, 下游的主要 出湖河流有位于东南部的太浦河, 太浦河的出水量占太湖总出水量的 $65 \%$ 左右 ${ }^{[17]}$. 由于缺乏所有站点的实 测资料, 根据水量平衡原理, 由 2014 年城东港、百渎口、大渲河、浯溪桥、梅梁湖百站、望亭站的实测资料以 及 2014 年的出湖总流量 (约为 $104 \times 10^{8} \mathrm{~m}^{3}$ ), 推算出长皇港以及太浦河的流量. 太湖是典型的风生流湖泊, 风场在水体循环中起很重要的作用, 为研究不同风向对太湖水体交换的影响,对 2014 年 NCEP 再分析风场 资料 (图 3) 进行分析后, 得出春、夏、秋、冬的常风向分别为 SE、ESE、NNE 和 N, 采用 1994-2014 年的多年平 均风速. 在太湖湖流人口处持续释放浓度为 $1 \mathrm{mg} / \mathrm{L}$ 的示踪剂, 在不改变流量的情况下分别对四季不同风向 作用下的水龄进行模拟计算,计算方案参见表 2 .

\begin{tabular}{|c|c|c|c|c|}
\hline 计算方案 & 风向 & 风速 $/(\mathrm{m} / \mathrm{s})$ & 染色剂人口(测站) & 出口(无染色剂测站) \\
\hline 1 & SE(春季) & 3.44 & \multirow{4}{*}{$\begin{array}{l}\text { 城东港、大渲河、浯溪桥、百渎 } \\
\text { 口、长览港(各方案相同) }\end{array}$} & \multirow{4}{*}{ 梅梁湖、太浦河 (各方案相同) } \\
\hline 2 & ESE(夏季) & 2.75 & & \\
\hline 3 & NNE(秋季) & 3.07 & & \\
\hline 4 & N(冬季) & 3.64 & & \\
\hline
\end{tabular}

表 2 计算方案

Tab. 2 Calculation setup 


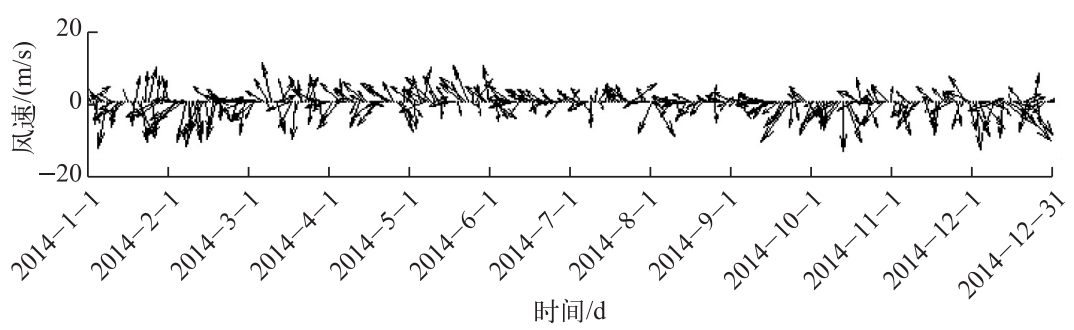

图 3 太湖风速矢量图

Fig.3 Wind velocity vector diagram of Lake Taihu

\section{3 水龄的计算以及模拟结果分析}

\section{1 染色剂和流场的季节性分布特征}

梅梁湖口的流速实测值在 $0.02 \sim 0.06 \mathrm{~m} / \mathrm{s}$ 之间 ${ }^{[17]}$, 本模型计算结果显示流速在 $0.03 \sim 0.07 \mathrm{~m} / \mathrm{s}$ 之间, 与实 测值吻合较好, 表明模型的精度满足要求. 染色剂和流场分布参见图 4, 由模拟结果可知, 湖流方向随风向的改 变而改变,染色剂从太湖人口处放人,随着湖流方向开始扩散. 春、夏偏南向季风作用下,竺山湖、西北湖区、西 南湖区的染色剂浓度较大; 秋、冬偏北向季风作用下,竺山湖、湖心区以及东部湖区的染色剂浓度较大.

\section{2 水龄季节性分布特征}

基于染色剂模型得出太湖水龄分布 (图 4). 结果表明, 染色剂浓度较大的地方水龄较小; 相反, 染色剂 浓度较小的地方水龄较大. 湖体水龄分布统计参见表 3. 春、夏、秋、冬季的湖体的平均水龄分别为 220.45 、 $235.62 、 217.47 、 211.80 \mathrm{~d}$. 秋、冬季的平均水龄小于春、夏季. 春、夏在偏南向季风作用下,竺山湖、西北湖区以 及西南湖区的水龄较小, $200 \mathrm{~d}$ 等水龄线分布范围较小; 秋、冬季在偏北风作用下, 竺山湖、梅梁湖、西南湖区 以及湖心区的水龄较小, 东部湖区、东太湖湖区的水龄较春、夏季有所减小, $200 \mathrm{~d}$ 等水龄线分布范围较大. 靠近湖流人口处的地方水龄较小, 远离人口的地方水龄较大; 水流流向与风向一致时水龄减小, 水循环加 快, 反之则减慢. 竺山湖是湖体水龄最小的地区, 贡湖则是湖体水龄最大的地区.

表 3 不同水龄区间 ( 天数) 所占湖区面积

Tab.3 The area covered by different water ages (days)

\begin{tabular}{|c|c|c|c|c|c|}
\hline \multirow{2}{*}{ 季节 } & \multicolumn{4}{|c|}{ 面积 $/ \mathrm{km}^{2}$} & \multirow{2}{*}{ 平均水龄/ $\mathrm{d}$} \\
\hline & $0 \sim 100 \mathrm{~d}$ & $100 \sim 200 \mathrm{~d}$ & $200 \sim 300 \mathrm{~d}$ & $300 \mathrm{~d}$ 以上 & \\
\hline 春季 & 422.36 & 366.02 & 714.10 & 575.00 & 220.45 \\
\hline 夏季 & 309.06 & 400.33 & 598.28 & 769.81 & 235.62 \\
\hline 秋季 & 433.69 & 450.68 & 554.53 & 638.57 & 217.47 \\
\hline 冬季 & 417.85 & 505.44 & 627.87 & 526.21 & 211.80 \\
\hline
\end{tabular}

\section{3 “引江济太”工程对水龄分布的影响}

每年太湖都有低水位的“枯水期”, 往年都是通过望虞河 “引江济太”, 确保太湖水位保持在 $3 \mathrm{~m}$ 以上. 为了更好地盘活太湖水体, 在从长江引水进人太湖的同时, 太湖流域内在 2014 年开始疏通新沟河、新孟河 两条河道, 太湖将通过新孟河、望虞河补水, 借助走马塘、新沟河、太浦河向长江排水, 太湖将形成 “两进三 出” 的循环系统, 通过让水体动起来, 改善水质. 为防止汛期太湖水位过高, 新沟河将承担太湖北部区域向长 江排水的功能. 同时,应急情况下也能通过新沟河向太湖调水,改善梅梁湖水质.

据实测资料分析, 2014 年新沟河和望虞河的引水时间并不在同一区间,2014 年新沟河在 6-8 月进行引 水 $20 \mathrm{~d}$, 共引水 $1.1522 \times 10^{8} \mathrm{~m}^{3}$, 而望虞河 2014 年 1-3 月、11-12 月共引水 $130 \mathrm{~d}$ (图 5), 引水量 $10.5759 \times$ $10^{8} \mathrm{~m}^{3}$. 据此本文对 2014 年望虞河引水和新沟河引水分别进行了计算对比, 在引水时放人 $1 \mathrm{mg} / \mathrm{L}$ 的染色 

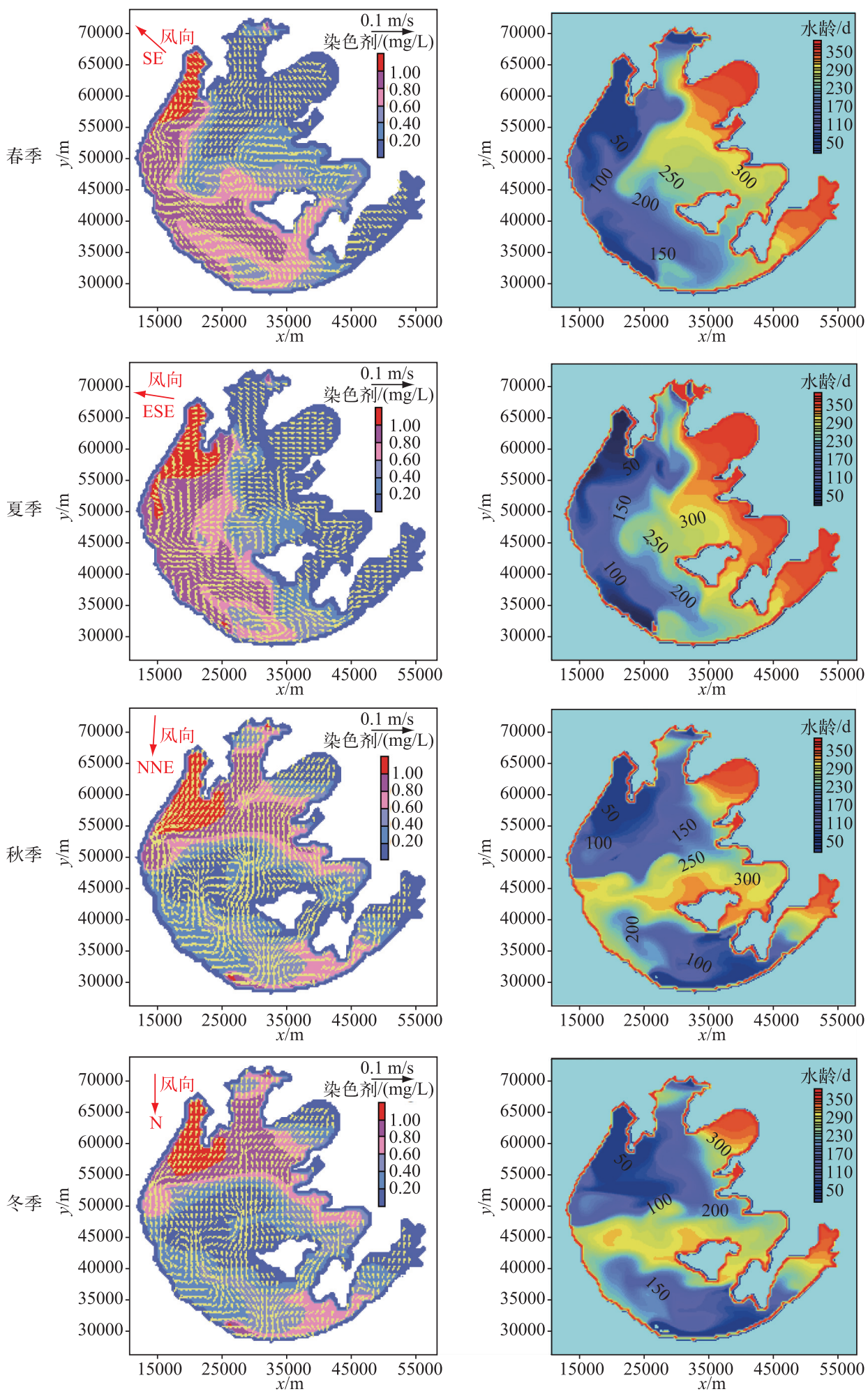

图 4 季风影响下太湖 4 个季节染色剂、流场和水龄分布

Fig.4 Distributions of dye, flow field and water age of Lake Taihu in each season 
剂, 其余湖流人口采用实测流量, 不放人染色剂, 通过染色剂的面积可以得出进人湖体的引水水体面积,进 一步分析对比从而评估引水的效益,模拟结果参见表 4 和图 6. 望虞河的引水量大于新沟河, 因此染色剂扩 散面积较大, 染色剂浓度大于 $0.01 \mathrm{mg} / \mathrm{L}$ 的面积约为新沟河的 5 倍; 染色剂大于 $0.001 \mathrm{mg} / \mathrm{L}$ 的面积约为太湖 总面积的 $1 / 4$, 因此可以看出望虞河引水效果良好, 表明引水量越大, 引水效果越好. 但是望虞河引水量约为 新沟河的 10 倍,染色剂面积未达到 10 倍, 是由于新沟河人口附近水体交换较快.

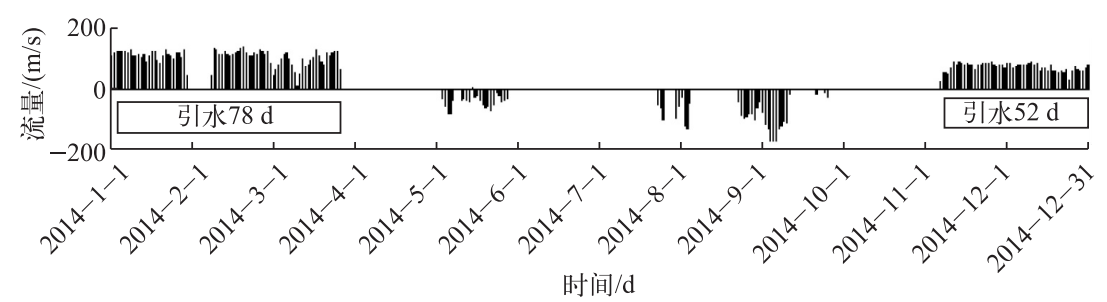

图 52014 年望亭站流量

Fig.5 Discharge of Wangting site in 2014
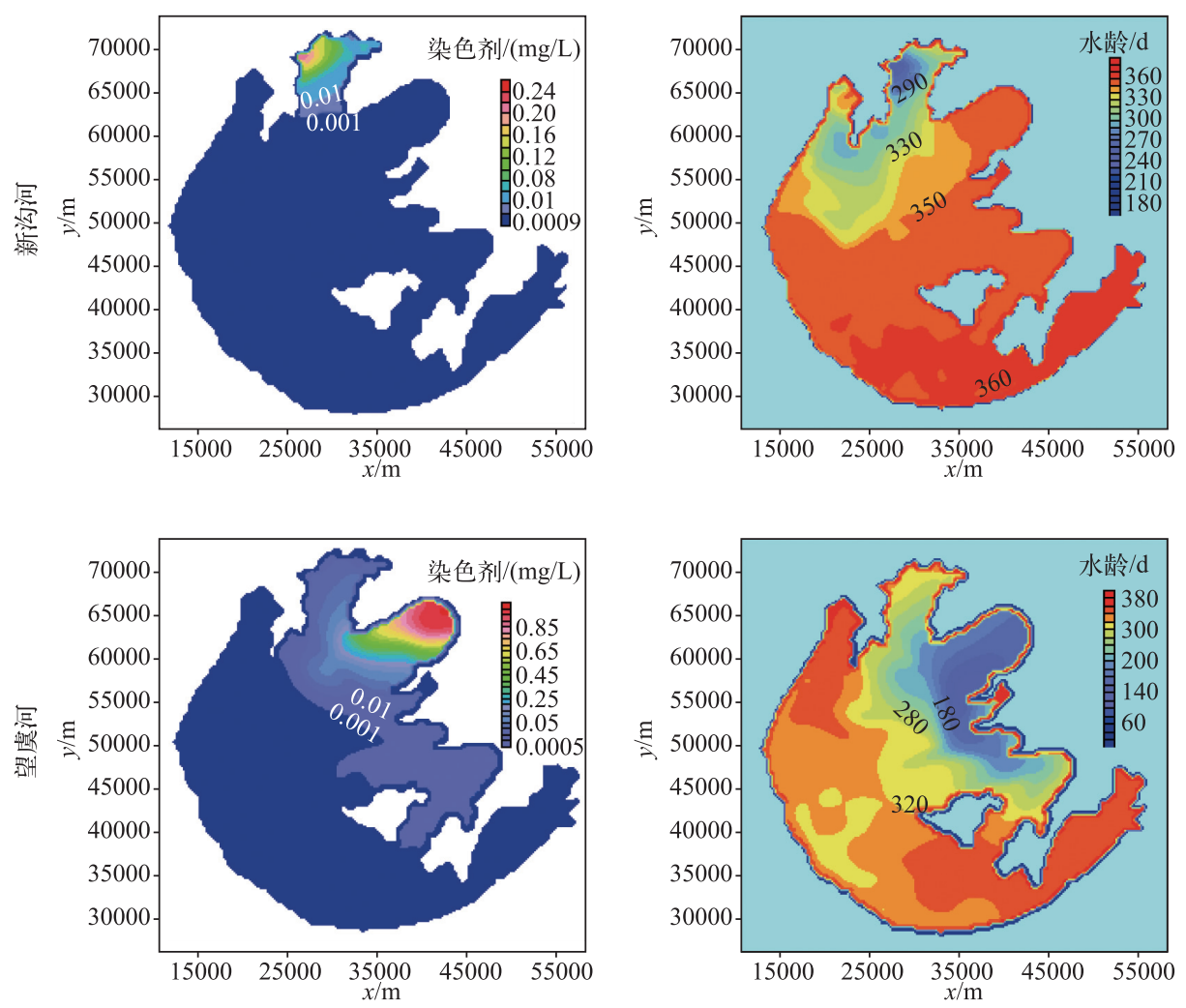

图 62014 年新沟河和望虞河引水运行条件下染色剂及水龄分布 (其余湖流不放人染色剂)

Fig.6 Distributions of dye and water age under operation of Xingou River and Wangyu River transfer in 2014 (other inlets with $0 \mathrm{mg} / \mathrm{L}$ dye)

为进一步评估引水方案对水龄的影响, 在对实际风场、流场下太湖的水龄进行计算的前提下, 分别在望 虞河和新沟河引水时也放人染色剂, 其余湖流人口采用实测流量, 均放人 $1 \mathrm{mg} / \mathrm{L}$ 染色剂, 模拟结果参见图 7 8. 结果表明望虞河引水工程可以加快贡湖的水体交换, 减小该地区的水龄; 从新沟河引水可以加快梅梁 湖地区的水体交换,改善梅梁湖的水质,但是由于引水量太小,因此对水龄的影响并不明显. 
表 4 染色剂分布面积

Tab.4 Distribution area of dye

\begin{tabular}{lccc}
\hline 引水河流 & 引水量 $/\left(\times 10^{8} \mathrm{~m}^{3}\right)$ & 染色剂浓度大于 $0.01 \mathrm{mg} / \mathrm{L}$ 的面积 $/ \mathrm{km}^{2}$ 染色剂浓度大于 $0.001 \mathrm{mg} / \mathrm{L}$ 的面积 $/ \mathrm{km}^{2}$ \\
\hline 新沟河 & 1.1522 & 67.04 & 84.35 \\
望虞河 & 10.5759 & 324.16 & 625.67 \\
\hline
\end{tabular}
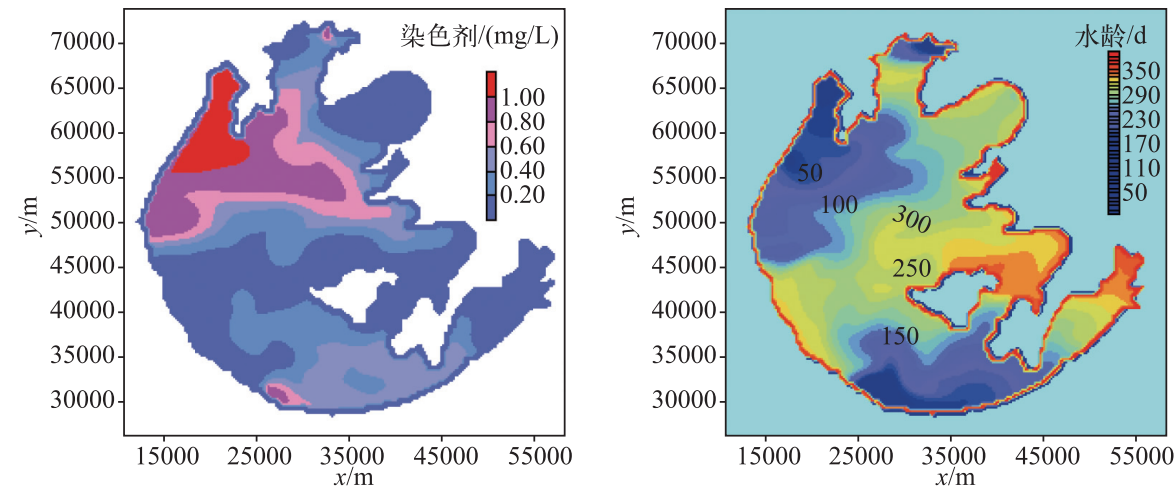

图 7 实际风场下染色剂和水龄分布 (其余湖流放人染色剂)

Fig.7 Distributions of dye and water age in actual wind field (other inlets with $1 \mathrm{mg} / \mathrm{L}$ dye)
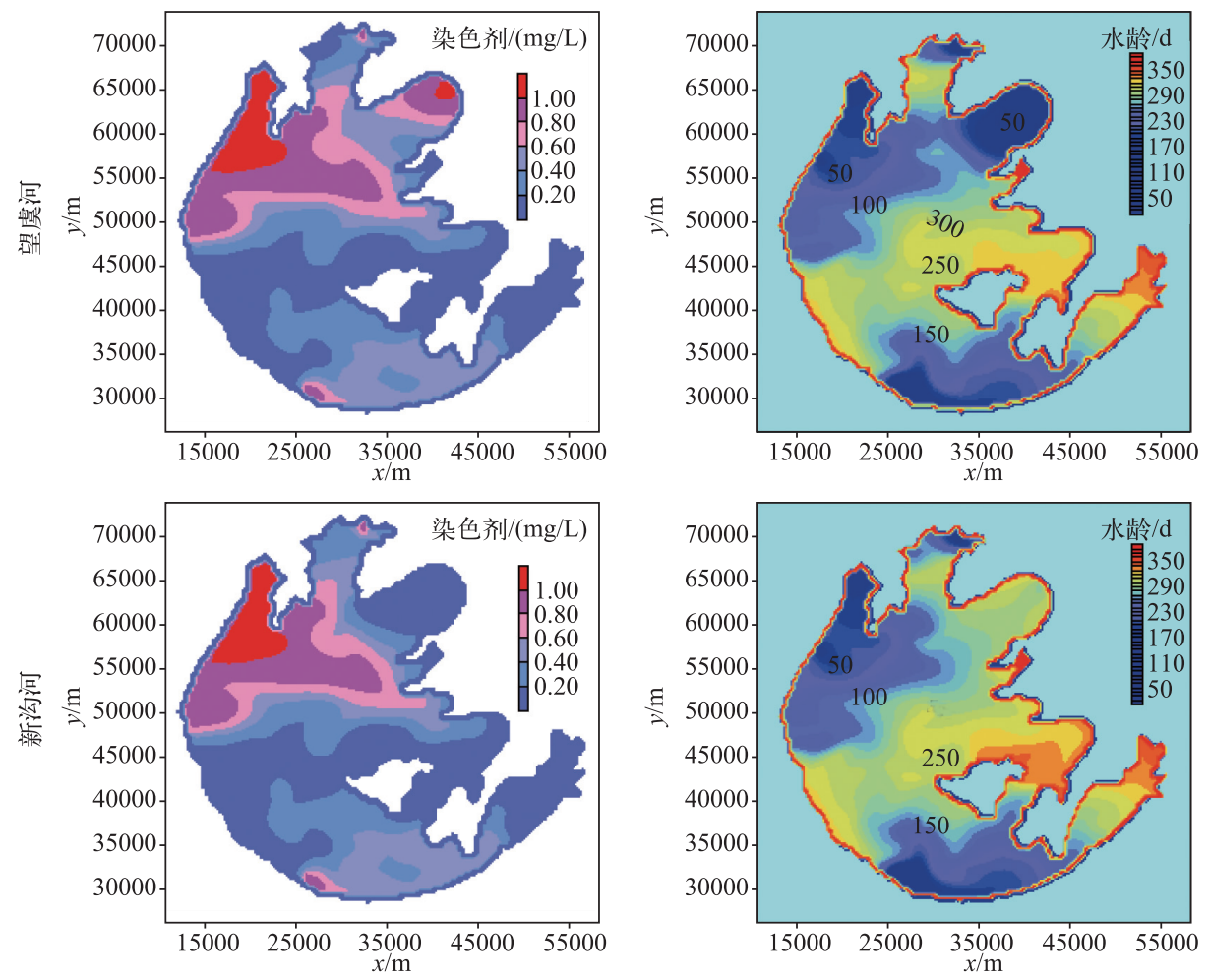

图 82014 年望虞河和新沟河引水运行条件下染色剂及水龄分布(其余湖流放人染色剂)

Fig. 8 Distributions of dye and water age under operation of Wangyu River and Xingou River transfer in 2014 (other inlets with $1 \mathrm{mg} / \mathrm{L}$ dye) 


\section{4 结论}

对于湖泊的水体交换评估, 水龄是一个非常重要的指标. 它反映了水质点从进人湖体到离开湖体的平 均时间,并受到流量、风场等因素的影响, 因此可以用来评估水体的更新速率. 本文建立了基于 EFDC 的三 维水动力模型来研究太湖水龄的分布以及 “引江济太”工程对其的影响. 春、夏季在偏南向季风作用下,竺山 湖、西北湖区以及西南湖区的水龄较小, $200 \mathrm{~d}$ 等水龄线分布范围较小; 秋、冬季在偏北风作用下, 竺山湖、梅 梁湖、西南湖区以及湖心区的水龄较小,东部湖区、东太湖湖区的水龄较春、夏季有所减小, $200 \mathrm{~d}$ 等水龄线 分布范围较大. 靠近人口处的地方水龄较小, 远离人口的地方水龄较大; 水流流向与风向一致时, 水龄减小, 水循环加快, 反之则减慢. 望虞河引水工程能够减小贡湖湖区的水龄, 加快该湖区及部分湖心区的水循环, 盘活太湖的东部地区,对太湖水污染的治理具有重要意义;而 2014 年新沟河工程采用的引水方案可以减小 梅梁湖区的水龄, 加快梅梁湖区的水循环, 改善梅梁湖水质, 并且通过流水带动, 改善人湖口直湖港、武进港 的水质. 在需要引水时, 新孟河、新沟河、望虞河均可引水, 可以组合使用, 以实现最优引水方案; 需要排水时 太浦河、新沟河、走马塘均可排水, 太浦河是主要通道. 引水工程的实施对加快整个太湖水体循环做出了重 要贡献.

致谢: 感谢江苏省水文水资源勘测局无锡分局的各位同仁在野外采样工作中给予的无私帮助.

\section{5 参考文献}

[ 1 ] Deleersnijder E, Campin JM, Delhez EJM. The concept of age in marine modelling I. Theory and preliminary model results. Journal of Marine Systems, 2001, 28(3/4) : 229-267.

[ 2 ] Li YP, Kumud A, Yu ZB. Modeling impacts of Yangtze River water transfer on water ages in Lake Taihu, China. Ecological Engineering, 2011, 37(2) : 325-334.

[ 3 ] Gao Yi, Mao Xinwei, Xu Weidong et al. Analysis of the influence on the Taihu Lake and the area around: Diversion from the Yangtze River to the Taihu Lake. Journal of China Hydrology, 2006, 26(1): 92-94. [高怡, 毛新伟, 徐卫东等. “引江济太”工程对太湖及周边地区的影响分析. 水文, 2006, 26(1) : 92-94.]

[ 4 ] Wu Haoyun. Study on key technologies of Yangtze-Taihu Water Diversion. China Water Resources, 2008, (1): 6-8. [吴浩 云. 引江济太调水试验关键技术研究和应用. 中国水利, 2008, (1) : 6-8.]

[ 5 ] Wu Haoyun, Zhou Danping, He Jia et al. Integrated benefit assessment of the project water diversion from Yangtze River to Lake Taihu and discussion on the methodology. J Lake Sci, 2008, 20(5) : 639-647. DOI: 10.18307/2008.0513. [吴浩 云, 周丹平, 何佳等. 引江济太工程综合效益的评估及方法探讨. 湖泊科学, 2008, 20(5) : 639-647.]

[6] Zhang Yifei, Wang Yulin, Wang Liang. EFDC overview and application analysis. Environmental Impact Assessment, 2015, (3) : 70-72, 92. [张以飞,王玉琳,汪鈽. EFDC 模型概述与应用分析. 环境影响评价, 2015, (3) : 70-72, 92.]

[ 7 ] Iglesias C, Mazzeo N, Meerhoff M et al. High predation is of key importance for dominance of small-bodied zooplankton in warm shallow lakes: Evidence from lakes, fish exclosures and surface sediments. Hydrobiologia, 2011, 667: 133-147.

[ 8 ] Ji ZG, Hu G, Shen J et al. Three-dimensional modeling of hydrodynamic processes in the St. Lucie Estuary. Estuarine, Coastal and Shelf Science, 2007, 73: 188-200.

[ 9 ] Lu Hui, Ning Yawei, Yuan Yongling. EFDC overview and application analysis. Water Resources and Power, 2013, (4): 100-102. [卢慧, 宁亚伟, 袁永龄. 基于 EFDC 模型的人工湖生态换水优化计算. 水电能源科学, 2013, (4): 100-102.]

[10] Li XW, Shen YM. Numerical simulation of the impacts of water level variation on water age in Dahuofang Reservoir. Frontiers in Earth Science, 2015, 9(2): 209-224.

[11] Deleersnijder E, Campin JM, Delhez EJM. The concept of age in marine modelling I. Theory and preliminary model results. Journal of Marine Systems, 2001, 28(3/4) : 229-267.

[12] Xiong Xiong. Hydrodynamic research of Poyang Lake based on the EFDC[Dissertation]. Nanchang: Nanchang University, 2014. [ 熊雄. 基于 EFDC 的鄱阳湖水动力研究 [学位论文]. 南昌: 南昌大学, 2014.]

[13] Shao Junrong, Wu Shiqiang, Zhou Jie et al. An age model for water transfer. Advances in Water Science, 2014, 25 ( 5) : 695-703. [邵军荣, 吴时强, 周杰等. 水体交换年龄模型研究. 水科学进展, 2014, 25(5) : 695-703.] 
[14] Liu Lüliu, Yang Zhifeng, Shen Zhenyao et al. Temporal characteristics of exchange and transit of water bodies. Journal of Natural Resources, 2003, 18(1):87-93. [刘绿柳, 杨志峰, 沈珍瑶等. 水体交换与传输的时间维特征. 自然资源学 报, 2003, 18(1): 87-93.]

[15] Qi Wen, Li Yiping, Wang Ying et al. Control and optimization scheme of TianJing Lake hydrodynamics based on EFDC Model. Water Resources and Power, 2015, (1): 47-51. [戚文, 李一平, 王莹等. 基于 EFDC 模型的天镜湖水动力优 化调控方案研究. 水电能源科学, 2015, (1): 47-51.]

[16] Gong Ran, Xu Jin, Xu Ligang et al. Study on hydrodynamics in urban landscape lake based on EFDC. Environmental Engineering, 2015, 33(4):58-62. [龚然, 徐进, 徐力刚等. 基于 EFDC 城市景观湖泊水动力模拟研究. 环境工程, $2015, \mathbf{3 3}(4): 58-62$.

[17] Qin Boqiang ed. Lake Taihu, China, dynamics and environmental change. Springer, 2008: 125. 